\title{
Small intestinal thrombotic microangiopathy following kidney transplantation diagnosed by balloon-assisted enteroscopy
}

\author{
Masafumi Nishio ${ }^{a}$, Kingo Hirasawaa , Jun-ichi Teranishi ${ }^{b}$, Koki Maedac, Yuichiro Ozekia , Atsushi Sawada ${ }^{a}$, \\ Ryosuke Ikeda ${ }^{a}$, Takehide Fukuchi ${ }^{a}$, Ryosuke Kobayashia, Makomo Makazua, Chiko Sato ${ }^{a}$, \\ Yoshiaki Inayamac, Shin Maedad
}

Yokohama City University Graduate School of Medicine, Yokohama, Japan

Abstract

\section{Introduction}

Thrombotic microangiopathy (TMA) is a serious complication following renal transplantation and causes hemolytic anemia, thrombocytopenia and organ injury [1]. Intestinal TMA (iTMA) is one of the organ disorders caused by TMA and causes abdominal pain, diarrhea and bloody stools. When the laboratory criteria for TMA are not met, histological

${ }^{a}$ Division of Endoscopy (Masafumi Nishio, Kingo Hirasawa, Yuichiro Ozeki, Atsushi Sawada, Ryosuke Ikeda, Takehide Fukuchi, Ryosuke Kobayashi, Makomo Makazu, Chiko Sato); ${ }^{b}$ Departments of Urology and Renal Transplantation (Jun-ichi Teranishi); ' Division of Diagnostic Pathology (Koki Maeda, Yoshiaki Inayama); ${ }^{\mathrm{D}}$ Department of Gastroenterology (Shin Maeda), Yokohama City University Graduate School of Medicine, Yokohama, Japan

Conflict of Interest: None

Correspondence to: Kingo Hirasawa, Division of Endoscopy, Yokohama City University Medical Center, 4-57 Urafune-cho, Minamiku, Yokohama 232-0024, Japan, e-mail: kingo_h@yokohama-cu.ac.jp

Received 30 July 2020; accepted 9 September 2020; published online 7 December 2020

DOI: https://doi.org/10.20524/aog.2020.0561 evaluation by endoscopic biopsy is necessary for the diagnosis of iTMA [2-4]. In recent years, various small intestinal diseases have been revealed by balloon-assisted enteroscopy (BAE), including double- and single-balloon enteroscopy. However, there are few reports of small intestinal TMA, and the clinical features and endoscopic findings remain unclear. Herein, we report a case of small intestinal TMA, following kidney transplantation, diagnosed by BAE.

\section{Case report}

A 32-year-old woman, who received a kidney transplant because of nephrotic syndrome at the age of 10 years, was admitted to our hospital with recurrent abdominal pain, diarrhea and bloody stools. Five months before admission, she was administered everolimus (EVE), a mammalian target of rapamycin (mTOR) inhibitor, in addition to tacrolimus and methylprednisolone for rejection prophylaxis, and her symptoms developed 1 month after the start of EVE administration. She received antibacterial therapy, but her symptoms did not improve. On admission, initial laboratory findings revealed mild anemia, thrombocytopenia, and slightly 
elevated lactate dehydrogenase (LDH), while schistocytes were not observed (Table 1). Thus, laboratory findings did not show any evidence of TMA. Further, no evidence of an infection (i.e., cytomegalovirus or Epstein-Barr virus) was found. Plain computed tomography of the abdomen showed wall thickening of the small intestine and ascites; there was therefore no evidence of organ disorders other than of the digestive tract. Intestinal ultrasound examination showed wall thickening with increased blood flow in the small intestine (Fig. 1). In contrast, esophagogastroduodenoscopy (EGD) and colonoscopy results were within normal limits. Subsequently, double-balloon enteroscopy (DBE) was performed, which revealed a circumferential ulcer over $20 \mathrm{~cm}$ in length in the jejunum. Enteroclysis with gastrografin revealed a small intestinal stricture that coincided with the site of the ulcer (Fig. 2A,B). Histopathological findings of the small intestine biopsy specimen revealed shortened villi, hyperemia in the mucosa, and thrombi within small vessels in the submucosa,

Table 1 Laboratory findings on admission

\begin{tabular}{lcc}
\hline Parameter & Patient & Reference \\
\hline Hematology & 10.0 & $11.6-14.8$ \\
\hline Hemoglobin $(\mathrm{g} / \mathrm{dL})$ & 112 & $158-348$ \\
\hline Platelet count $\left(\times 10^{3} / \mu \mathrm{L}\right)$ & 14.8 & $3.3-8.6$ \\
\hline White blood cell count $\left(\times 10^{3} / \mu \mathrm{L}\right)$ & $<0.1$ & \\
\hline Schistocyte count & & \\
\hline Blood chemistry & 284 & $124-222$ \\
\hline Lactate dehydrogenase $(\mathrm{U} / \mathrm{L})$ & 2.1 & $4.1-5.1$ \\
\hline Albumin $(\mathrm{g} / \mathrm{dL})$ & 4.18 & $0.46-0.79$ \\
\hline Serum creatinine $(\mathrm{mg} / \mathrm{dL})$ & 0.4 & $0.4-1.5$ \\
\hline Serum bilirubin $(\mathrm{mg} / \mathrm{dL})$ & 6.6 & $<0.14$ \\
\hline C-reactive protein $(\mathrm{mg} / \mathrm{dL})$ & & \\
\hline
\end{tabular}

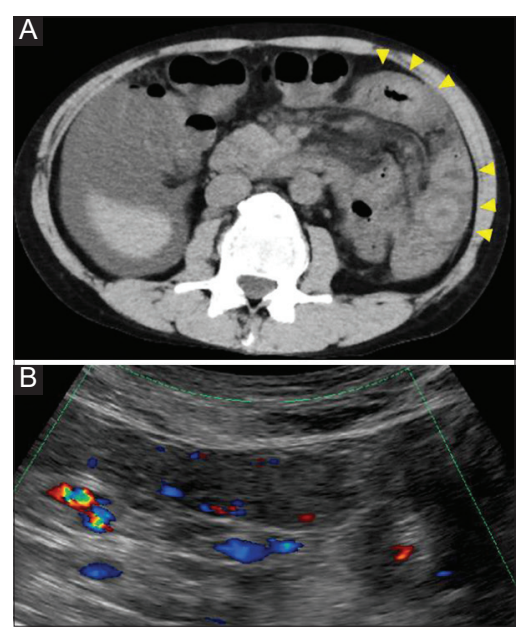

Figure 1 (A) Abdominal computed tomography shows wall thickening of the small intestine (yellow arrowheads) and ascites. No free air is found. (B) Abdominal ultrasound shows wall thickening with focally increased Doppler flow leading to the diagnosis of small intestinal TMA (Fig. 3). Considering the clinical course, with the symptoms of the patient appearing after EVE administration, she was diagnosed with EVE-induced TMA. Her symptoms, consisting of abdominal pain, diarrhea and bloody stools, improved after the discontinuation of EVE. Ulcer healing was observed by DBE 4 months after the discontinuation of EVE (Fig. 2C).

\section{Discussion}

Two key findings should be highlighted in this case. First, although the laboratory findings did not meet the TMA diagnostic criteria [5], a definitive diagnosis was achieved by performing DBE. In the criteria proposed by the European Group for Blood and Marrow Transplantation, TMA is defined as a syndrome with the following laboratory findings: i) hemolytic anemia; ii) thrombocytopenia; iii) a schistocyte count of $>4 \%$; iv) increased $\mathrm{LDH}$; and v) decreased haptoglobin [5]. In the present case, thrombocytopenia was the only TMA criterion that was met. The clinical symptoms of iTMA are reported as abdominal pain, diarrhea and bloody stools, difficult to distinguish from graft-versus-host disease, an infection, and ischemic enteritis/colitis. On the other hand, previous studies have reported that some patients have been diagnosed with iTMA histologically, even if their laboratory findings do not meet the TMA criteria [2-4]. In such cases, histological investigation by endoscopic biopsy is important for the diagnosis of iTMA. To the best of our knowledge, the present report is the first iTMA case confined to the small intestine that could not be diagnosed by EGD and colonoscopy alone, but additionally required DBE. In recent years, BAE has become popular in many countries, but there have been no reports of small intestinal TMA diagnosed by BAE. The endoscopic findings of TMA in the duodenum and colon have been reported as edema, redness and ulcers [6,7], but since there have been only a limited number of reports, the endoscopic characteristics of iTMA have been unclear. The present case showed circumscribed and extensive ulcers, which were not found in the duodenum and colorectal lesions in previous reports. Given that cases of intestinal perforation due to iTMA have been reported, the early diagnosis of iTMA is important. However, since the risk of bleeding, perforation and aspiration pneumonia may be elevated in patients who are in poor general condition, the indication for BAE should be carefully considered. This case suggests that BAE is a useful tool for diagnosing TMA confined to the small intestine when a histopathological diagnosis is imperative, rather than a necessary procedure that should be performed in every posttransplant patient with bloody diarrhea and abdominal pain.

The second key finding to highlight is that EVE can cause intestinal TMA. mTOR inhibitors, including sirolimus and EVE, are known to cause TMA after kidney transplantation [1]. Previous studies have demonstrated that the risk of TMA is significantly increased in patients who receive a combination of $\mathrm{mTOR}$ inhibitors and calcineurin inhibitors, including tacrolimus and cyclosporine, compared 

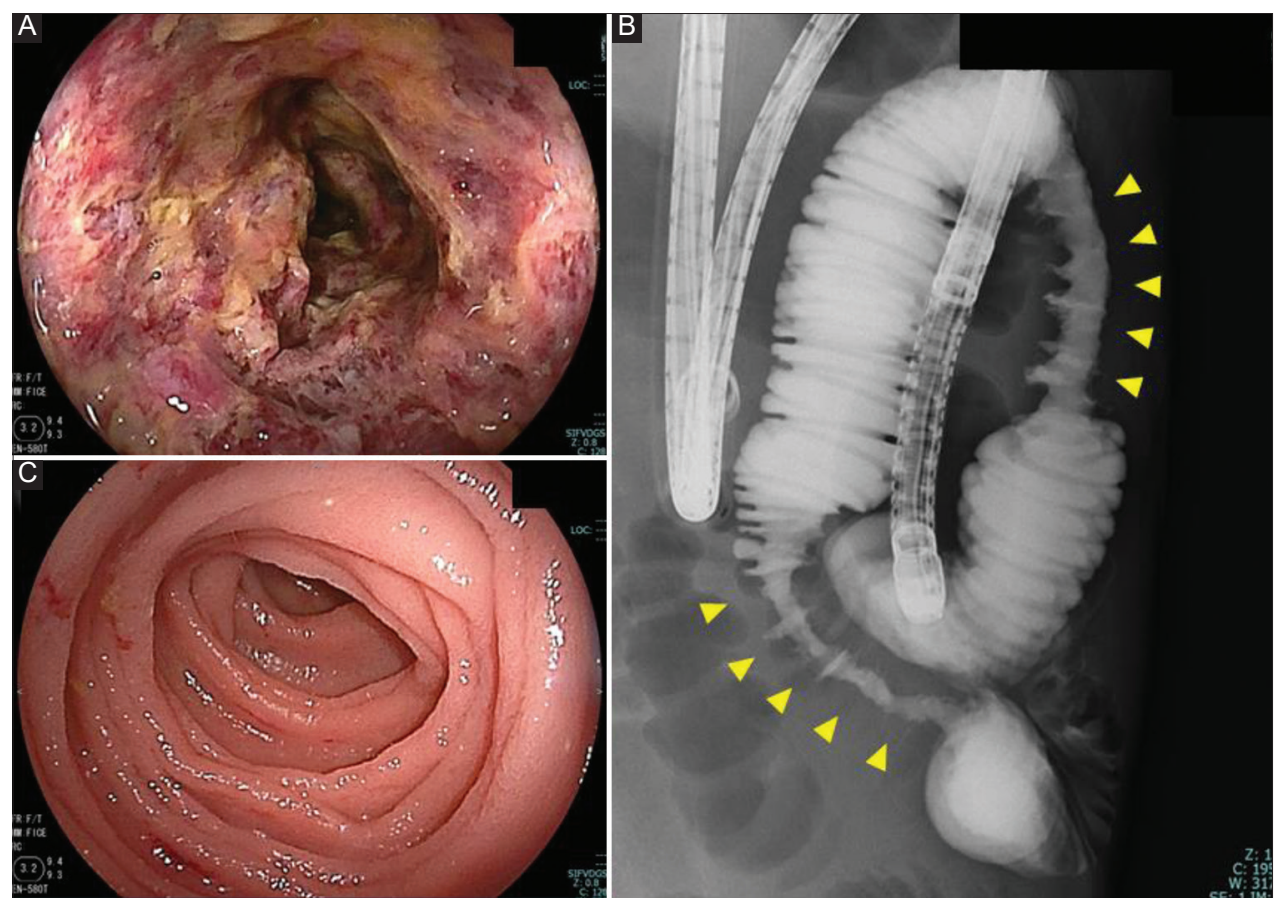

Figure 2 (A) Endoscopic view of the small intestine on admission. A severe circumferential ulcer measuring over $20 \mathrm{~cm}$ in length was found in the jejunum. (B) Enteroclysis with gastrografin revealed a small intestinal stenosis at the ulcer site. Two stricture sites in the jejunum were skipped (yellow arrowheads). (C) Endoscopic view of the small intestine 4 months after the discontinuation of everolimus. The severe circumferential ulcer has healed without stricture

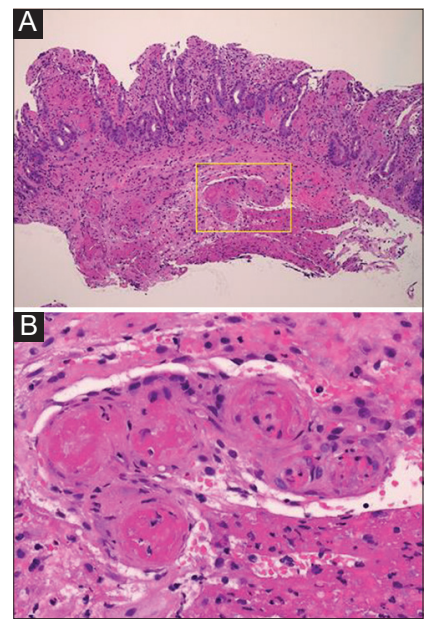

Figure 3 (A) Histopathological findings of the small intestinal biopsy specimen with hematoxylin and eosin staining revealed shortened villi and hyperemia in the mucosa. (B) Detail of the yellow box in (a); histopathological findings revealed thrombi within some small vessels in the submucosa, compatible with intestinal thrombotic microangiopathy

to receiving either medication alone [8]. The initial treatment for drug-induced TMA is the discontinuation of the causative drug, and in the present case, iTMA was improved by the discontinuation of EVE. Eculizumab has been reported to be effective if improvement in TMA is not achieved by the discontinuation of the causative drug [9]. Although a previous study reported sirolimus-induced iTMA [10], there have been no reports of EVE-induced iTMA. Thus, to the best of our knowledge, the present case is also the first report of EVEinduced iTMA.

In conclusion, BAE is useful for diagnosing TMA confined to the small intestine when a histopathological diagnosis is imperative, rather than a necessary procedure that should be performed in every post-transplant patient with bloody diarrhea and abdominal pain. Furthermore, EVE may be the cause of small intestinal TMA. The present case suggests that BAE should be performed in patients taking immunosuppressive medication following kidney transplantation, who complain of abdominal pain, diarrhea and bloody stools, and yet have no abnormal EGD or colonoscopy findings, even if there is no evidence of TMA as defined by laboratory findings. Further accumulation of cases is needed to clarify the clinical course and endoscopic characteristics of small intestinal TMA.

\section{References}

1. Garg N, Rennke HG, Pavlakis M, Zandi-Nejad K. De novo thrombotic microangiopathy after kidney transplantation. Transplant Rev (Orlando) 2018;32:58-68.

2. Nishida $\mathrm{T}$, Hamaguchi $\mathrm{M}$, Hirabayashi $\mathrm{N}$, et al. Intestinal thrombotic microangiopathy after allogeneic bone marrow transplantation: a clinical imitator of acute enteric graft-versushost disease. Bone Marrow Transplant 2004;33:1143-1150.

3. Narimatsu $H$, Kami $M$, Hara $S$, et al. Intestinal thrombotic microangiopathy following reduced-intensity umbilical cord blood 
transplantation. Bone Marrow Transplant 2005;36:517-523.

4. Inamoto $\mathrm{Y}$, Ito $\mathrm{M}$, Suzuki $\mathrm{R}$, et al; Nagoya Blood and Marrow Transplantation Group. Clinicopathological manifestations and treatment of intestinal transplant-associated microangiopathy. Bone Marrow Transplant 2009;44:43-49.

5. Ruutu T, Barosi G, Benjamin RJ, et al; European LeukemiaNet. Diagnostic criteria for hematopoietic stem cell transplantassociated microangiopathy: results of a consensus process by an International Working Group. Haematologica 2007;92:95-100.

6. Yamamoto S, Nakase H, Chiba T, Yamashita K, Ito M. Clinical challenges and images in GI. Image 4. Transplant-associated thrombotic microangiopathy of the colon accompanied by graftversus-host disease. Gastroenterology 2009;136:2067, 2418.

7. Nunius C, Büttner-Herold M, Bertz S, Schiffer M, Buchholz B.
Isolated thrombotic microangiopathy of the small intestine in a patient with atypical hemolytic uremic syndrome - a case report. BMC Nephrol 2020;21:104.

8. Fortin MC, Raymond MA, Madore F, et al. Increased risk of thrombotic microangiopathy in patients receiving a cyclosporinsirolimus combination. Am J Transplant 2004;4:946-952.

9. Epperla N, Hemauer K, Hamadani M, Friedman KD, Kreuziger LB. Impact of treatment and outcomes for patients with posttransplant drug-associated thrombotic microangiopathy. Transfusion 2017;57:2775-2781.

10. Robson M, Côte I, Abbs I, Koffman G, Goldsmith D. Thrombotic micro-angiopathy with sirolimus-based immunosuppression: potentiation of calcineurin-inhibitor-induced endothelial damage? Am J Transplant 2003;3:324-327. 